\title{
CONTINUOUS IMAGES OF ARCS
}

\author{
JACEK NIKIEL
}

(Communicated by Dennis K. Burke)

\begin{abstract}
A characterization of continuous images of (Hausdorff) arcs is used to describe their cyclic elements as inverse limits of inverse systems of 'nice' spaces with 'nice' bonding mappings.
\end{abstract}

Introduction. By a continuum we will mean a compact and connected Hausdorff space, and an arc will be understood as a Hausdorff arc, i.e., a continuum with exactly two non-cut-points. Recall that arcs are precisely compact and connected linearly ordered topological spaces, and each separable arc is homeomorphic to $[0,1]$ (see for example [6]).

The classical Hahn-Mazurkiewicz theorem $[5,10]$ characterizes continuous images of $[0,1]$ (in the class of all Hausdorff spaces) as locally connected and metrizable continua. Since 1960 it is known that there exist locally connected continua which are continuous images of no arc ([7]; recall that each Hausdorff space which is a continuous image of some arc is a locally connected continuum - see for example [6]). Hence there is no straightforward generalization of the Hahn-Mazurkiewicz theorem. The first reasonable characterization of continuous images of arcs was found in 1985 in [12], where several natural equivalent conditions were proved. Some of those conditions involve the use of the cyclic element theory (see for example [20] for the cyclic element theory in metrizable locally connected continua, and $[21,2]$ for generalizations of the theory to the class of all locally connected continua). The utility of the cyclic element theory as a way to find a nonseparable version of the Hahn-Mazurkiewicz theorem was noticed for the first time in [2], where it was proved that if each cyclic element of a locally connected continuum $X$ is a continuous image of some arc, then also the whole $X$ is an image of some arc.

In the present paper we apply the characterization theorem of [12] to prove that if $X$ is a cyclic element of a continuum which is a continuous image of some arc, then $X$ is homeomorphic to the inverse limit of an inverse sequence of some 'piecewise' metrizable continua (each of which is also a continuous image of some arc and, moreover, can be defined by a simple induction) with natural bonding mappings. Moreover, we describe some examples and applications of the obtained results. Some further applications of the related methods can be found in [14].

2. Auxiliary facts. Let $X$ be a locally connected continuum. We say that a subset $A$ of $X$ is a $T$-set in $X$ provided $A$ is a closed set and each component

Received by the editors November 21, 1986 and, in revised form May 7, 1987.

1980 Mathematics Subject Classification (1985 Revision). Primary 54B25, 54F25; Secondary $54 \mathrm{C} 10$.

Key words and phrases. Arc, cyclic element, Hahn-Mazurkiewicz theorem, inverse limit, monotone mapping. 
of $X-A$ has a two-point boundary. If $X$ has no cut points, then we say that a sequence $A_{1}, A_{2}, \ldots$ of $T$-subsets of $X T$-approximates $X$ provided

(1) $A_{1} \subset A_{2} \subset \cdots$,

(2) if $P$ is a component of $X-A_{n}, n=1,2, \ldots$, then the set of all cut points of $\bar{P}$ is contained in $A_{n+1}$,

(3) if $P$ is a component of $X-A_{n}, n=1,2, \ldots$, and $C$ is a nondegenerate cyclic element of $\bar{P}$, then the set $C \cap A_{n+1}$ is metrizable and contains at least three points, and

(4) $A_{1}$ is metrizable.

It turns out that if $X$ has no cut points and a sequence $A_{1}, A_{2}, \ldots$ of $T$-subsets of $X$ fulfills (1), (2) and

$\left(3^{\prime}\right)$ if $P$ is a component of $X-A_{n}, n=1,2, \ldots$, and $C$ is a nondegenerate cyclic element of $\bar{P}$, then the set $C \cap A_{n+1}$ contains at least three points, then $A=\bigcup_{n=1}^{\infty} A_{n}$ is a dense subset of $X$ (see [12, Lemma 3.4]).

Theorem 2.1. is a particular case of [12, Theorem 1.1]:

2.1. THEOREM. If $X$ is a continuum, then the following conditions are equivalent:

(i) $X$ is a continuous image of some arc,

(ii) $X$ is locally connected and if $Y$ is a nondegenerate cyclic element of $X$ and $p, q, r \in Y$ are any points, then there is a metrizable $T$-set $A$ in $Y$ such that $p, q, r \in A$,

(iii) $X$ is locally connected and each nondegenerate cyclic element $Y$ of $X$ can be $T$-approximated by some sequence $A_{1}^{Y}, A_{2}^{Y}, \ldots$ of $T$-subsets of $Y$.

Let $X$ and $Y$ be locally connected continua, $f: X \rightarrow Y$ be a function and $A$ be a $T$-set in $X$. We say that $f$ is a $T$-map with respect to $A$ provided

(5) $f$ is a continuous surjection,

(6) $B=f(A)$ is a $T$-set in $Y$ and $\left.f\right|_{A}$ is a homeomorphism from $A$ onto $B$,

(7) each component $Q$ of $Y-B$ is homeomorphic to ]0, 1[ (so $\bar{Q}$ is homeomorphic to $[0,1])$, and

(8) for each component $Q$ of $Y-B$ there is the unique component $P_{Q}$ of $X-A$ such that $f\left(P_{Q}\right) \subset \bar{Q}$, and each component of $X-A$ is a $P_{Q}$ for some component $Q$ of $Y-B$.

Furthermore, $f: X \rightarrow Y$ is said to be a $T$-map if it is a $T$-map with respect to some $T$-subset $A$ of $X$.

2.2. LEMMA. If $X$ is a locally connected continuum and $A$ is a $T$-set in $X$, then there are a locally connected continuum $X_{A}$ and a function $f_{A}: X \rightarrow X_{A}$ such that $f_{A}$ is a T-map with respect to $A$. Moreover, $X_{A}$ is determined uniquely up to a homeomorphism.

ProOF. The first part of Lemma 2.2 was shown by L. B. Treybig, [16, Theorem 6], for the second part see [12, Lemma 3.5].

2.3. LEMMA. If $X$ is a locally connected continuum, $A, B$ are $T$-sets in $X$ such that $B \subset A$, and $f: X \rightarrow X_{A}$ is a T-map with respect to $A$, then $f(B)$ is a $T$-set in $X_{A}$. Moreover, if $P$ is a component of $X-B$, then there is the unique component $Q$ of $X_{A}-f(B)$ such that $Q \subset f(P) \subset \bar{Q}$. 
ProOF. Let $P$ be a component of $X-B, \operatorname{bd}(P)=\left\{b_{1}, b_{2}\right\}$, and put $Q=$ $f(P)-f\left(\left\{b_{1}, b_{2}\right\}\right)$. It suffices to show that $Q$ is a component of $X_{A}-f(B)$ such that $\operatorname{bd}(Q)=f\left(\left\{b_{1}, b_{2}\right\}\right)$. Note that

$$
f^{-1} f\left(b_{i}\right) \subset\left\{b_{i}\right\} \cup \bigcup\left\{U: U \text { is a component of } X-A \text { and } b_{i} \in \operatorname{bd}(U)\right\} \text {. }
$$

The set $G=(X-P) \cup f^{-1} f\left(\left\{b_{1}, b_{2}\right\}\right)$ is closed in $X$. Moreover, $Q=X_{A}-f(G)$ (because $f$ is a $T$-map and $B \subset A)$. Hence $Q$ is an open subset of $X_{A}$. Observe that $\operatorname{bd}(Q)=f\left(\left\{b_{1}, b_{2}\right\}\right)$. Suppose that $Q$ is not connected and let $V_{1}, V_{2}$ be nonempty, disjoint and open subsets of $Q$ such that $Q=V_{1} \cup V_{2}$. Put

$$
W_{i}=\left(f^{-1}\left(V_{i}\right) \cap A\right) \cup \bigcup\left\{U: U \text { is a component of } X-A \text { and } f(U) \cap V_{i} \neq \varnothing\right\} \text {, }
$$

for $i=1,2$. Note that $W_{1}, W_{2}$ are nonempty, disjoint and open subsets of $P$ such that $P=W_{1} \cup W_{2}$. Hence $P$ is not connected, a contradiction.

2.4. LEMMA. If $X$ is a continuum without cut points which is a continuous image of some arc, $A_{1}, A_{2}, \ldots$ is a sequence of $T$-subsets of $X$ which $T$-approximates $X$, and $f_{n}: X \rightarrow X_{A_{n}}$ is a $T$-map with respect to $A_{n}$, for $n=1,2, \ldots$, then the family $\left\{f_{1}, f_{2}, \ldots\right\}$ separates points of $X$.

Proof. Put $A=\bigcup_{n=1}^{\infty} A_{n}$; so $A$ is dense in $X$. Let $x$ and $y$ be distinct points of $X$. We consider three cases.

Case 1. $x, y \in A$. Hence there is an integer $n$ such that $x, y \in A_{n}$. Then $f_{n}(x) \neq f_{n}(y)$.

Case 2. $x \in A$ and $y \notin A$. By [16, Theorem 8], there is an integer $n$ such that $x \in A_{n}$ and $x \notin \operatorname{bd}(P)$, where $P$ is a component of $X-A_{n}$ such that $y \in P$. Therefore $f_{n}(x) \neq f_{n}(y)$.

Case 3. $x, y \notin A$. By [16, Theorem 8] there is an integer $n$ such that if $P, Q$ are components of $X-A_{n}$ so that $x \in P, y \in Q$, then $\operatorname{bd}(P) \cap \operatorname{bd}(Q)=\varnothing$. Hence $f_{n}(x) \neq f_{n}(y)$.

3. The main result. Let $X$ be a continuum which is a continuous image of some arc (so $X$ is locally connected). We say that the rank of $X$ is less than or equal to a nonnegative integer $m, r(X) \leq m$, provided either

(9) each cyclic element of $X$ is degenerate or

(10) $X$ has a nondegenerate cyclic element and for each nondegenerate cyclic element $Y$ of $X$ there is a sequence $A_{1}^{Y}, A_{2}^{Y}, \ldots$ of $T$-subsets of $Y$ which $T$ approximates $Y$ and is such that $A_{m}^{Y}=Y$.

We say that the rank of $X$ is equal to $m$ provided $r(X) \leq m$ and either $m=0$ or $r(X) \$ m-1$.

3.1. PROPOSITION. If $X$ is a continuum which is a continuous image of some arc, then

(i) $r(X)=0$ if and only if $X$ is a dendron,

(ii) $r(X) \leq 1$ if and only if each cyclic element of $X$ is metrizable.

3.2. THEOREM. Let $X$ be a continuum without cut points which is a continuous image of some arc. If $A_{1}, A_{2}, \ldots$ is a sequence of $T$-subsets of $X$ which $T$-approximates $X$, then

(i) $r\left(X_{A_{n}}\right) \leq n$ for $n=1,2, \ldots$, and 

that

(ii) there are functions $f_{n}: X \rightarrow X_{A_{n}}$ and $g_{n}: X_{A_{n+1}} \rightarrow X_{A_{n}}, n=1,2, \ldots$, such

(a) each $f_{n}$ is a $T$-map with respect to $A_{n}$,

(b) each $g_{n}$ is a T-map with respect to $f_{n+1}\left(A_{n}\right)$, and

(c) the diagrams

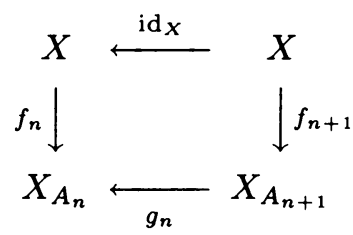

commute, and so $X$ is homeomorphic to $\lim \operatorname{inv}\left(X_{A_{n+1}}, g_{n}\right)$.

PROOF. For simplicity, we will write $X_{n}$ instead of $X_{A_{n}}$.

(i) By Lemma $2.3, S=\left\{f_{n}\left(A_{1}\right), f_{n}\left(A_{2}\right), \ldots, f_{n}\left(A_{n-1}\right), X_{n}\right\}$ is a sequence of $T$-subsets of $X_{n}$. To see that $S T$-approximates $X_{n}$ it suffices to prove that if $P$ is a component of $X_{n}-f_{n}\left(A_{n-1}\right)$ and $C$ is a nondegenerate cyclic element of $\bar{P}$, then the set $C \cap X_{n}=C$ is metrizable. Let $Q$ denote the unique component of $X-A_{n-1}$ such that $f_{n}(Q) \subset \bar{P}$. There is the unique cyclic element $D$ of $\bar{Q}$ such that $f_{n}(D)=C$. Note that $\left.f_{n}\right|_{D}: D \rightarrow C$ is a $T$-map with respect to $A_{n} \cap D$ and $A_{n} \cap D$ is metrizable. By [12, Lemma 4.2], $C$ is metrizable.

(ii) Note that, by [12, Lemma 4.2], $X_{1}$ is a metrizable space. Let $h_{1}: X \rightarrow X_{1}$ be any $T$-map with respect to $A_{1}$. Suppose that, for some positive integer $n$, the function $h_{n}: X \rightarrow X_{1}$ is already constructed such that $h_{n}$ is a $T$-map with respect to $A_{1},\left.h_{n}\right|_{A_{1}}=\left.h_{1}\right|_{A_{1}}$, and for each component $P$ of $X-A_{n}, \operatorname{bd}(P)=\left\{p_{1}, p_{2}\right\}$, the set $h_{n}(\bar{P})$ is an arc (maybe degenerate) with endpoints $h_{n}\left(p_{1}\right), h_{n}\left(p_{2}\right)$.

Let $x$ be any point of $X$. If $x \in A_{n+1}$, then define $h_{n+1}(x)=h_{n}(x)$. Suppose that $x \notin A_{n+1}$ and let $P$ denote a component of $X-A_{n+1}$ such that $x \in P$, $\operatorname{bd}(P)=\left\{p_{1}, p_{2}\right\}$. Let $Q$ be a component of $X-A_{1}$ such that $P \subset Q, \operatorname{bd}(Q)=$ $\left\{q_{1}, q_{2}\right\}$. Recall that $I=h_{n}(\bar{Q})$ is an arc with endpoints $h_{n}\left(q_{1}\right), h_{n}\left(q_{2}\right)$. We may assume that $h_{n}\left(q_{1}\right) \leq h_{n}\left(p_{1}\right) \leq h_{n}\left(p_{2}\right) \leq h_{n}\left(q_{2}\right)$ in the fixed natural ordering of $I$. Define

$$
h_{n+1}(x)= \begin{cases}h_{n}(x) & \text { if } h_{n}\left(p_{1}\right) \leq h_{n}(x) \leq h_{n}\left(p_{2}\right) \\ h_{n}\left(p_{1}\right) & \text { if } h_{n}(x) \leq h_{n}\left(p_{1}\right) \\ h_{n}\left(p_{2}\right) & \text { if } h_{n}\left(p_{2}\right) \leq h_{n}(x) .\end{cases}
$$

It is obvious that $h_{n+1}$ is a $T$-map with respect to $A_{1}$ (the continuity of $h_{n+1}$ follows from Lemma 3.1 of $[\mathbf{1 2}])$.

Note that the sequence $h_{1}, h_{2}, \ldots$ uniformly converges to a function $f_{1}: X \rightarrow X_{1}$ (in the fixed metric on $X_{1}$; use for example the second part of Lemma 3.4 of [12]). Moreover, $f_{1}$ is a $T$-map with respect to $A_{1}$, and

for each positive integer $m$ and each component $P$ of $X-A_{m}$,

$(* 1) \quad \operatorname{bd}(P)=\left\{p_{1}, p_{2}\right\}$, the set $f_{1}(\bar{P})$ is an arc (maybe degenerate) from $f_{1}\left(p_{1}\right)$ to $f_{1}\left(p_{2}\right)$.

Suppose that for some positive integer $n$ the function $f_{n}: X \rightarrow X_{n}$ is already constructed such that $f_{n}$ is a $T$-map with respect to $A_{n}$, and

for each positive integer $m, m \geq n$, and each component $P$ of $X-$

$(* n) \quad A_{m}, \operatorname{bd}(P)=\left\{p_{1}, p_{2}\right\}$, the set $f_{n}(\bar{P})$ is an arc (maybe degenerate) from $f_{n}\left(p_{1}\right)$ to $f_{n}\left(p_{2}\right)$. 
We define functions $f_{n+1}: X \rightarrow X_{n+1}$ and $g_{n}: X_{n+1} \rightarrow X_{n}$ such that $f_{n+1}$ is a $T$-map with respect to $A_{n+1}$ so that $(* n+1)$ holds, $g_{n}$ is a $T$-map with respect to $f_{n+1}\left(A_{n}\right)$, and $f_{n}=g_{n} \circ f_{n+1}$.

Let $i: X \rightarrow X_{n+1}$ be any $T$-map with respect to $A_{n+1}$. If $x \in A_{n+1}$ then define $f_{n+1}(x)=i(x)$ and if $y \in i\left(A_{n+1}\right), y=i(x)$ for some $x \in A_{n+1}$, then set $g_{n}(y)=f_{n}(x)$. Let $x$ be any point of $X-A_{n+1}$ and let $P$ denote a component of $X-A_{n+1}$ such that $x \in P, \operatorname{bd}(P)=\left\{p, p^{\prime}\right\}$. Let also $Q$ be a component of $X_{n+1}-i\left(A_{n+1}\right)$ such that $i(P) \subset \bar{Q}$. Recall that $\operatorname{bd}(Q)=\left\{i(p), i\left(p^{\prime}\right)\right\}$ and $\bar{Q}$ is homeomorphic to $[0,1]$. If $f_{n}(P)$ consists of a single point $z$, then put $f_{n+1}(x)=i(x)$ and $g_{n}(i(x))=z$. Assume that $f_{n}(P)$ is nondegenerate. By $(* n)$, $f_{n}(\bar{P})$ is a metrizable arc with endpoints $f_{n}(p), f_{n}\left(p^{\prime}\right)$. Let $j: \bar{Q} \rightarrow f_{n}(\bar{P})$ be any homeomorphism such that $j(i(p))=f_{n}(p)$ and put $f_{n+1}(x)=j^{-1}(z)$ provided $z=f_{n}(x)$. Set also $g_{n}\left(f_{n+1}(x)\right)=j\left(f_{n+1}(x)\right)$.

Now, observe that $f_{n+1}: X \rightarrow X_{n+1}$ and $g_{n}: X_{n+1} \rightarrow X_{n}$ are well-defined functions. By Lemma 3.1 of $[\mathbf{1 2}]$, these functions are continuous. An easy straightforward proof shows that $f_{n+1}$ and $g_{n}$ fulfill all the required conditions.

Since $f_{n}=g_{n} \circ f_{n+1}$, for $n=1,2, \ldots$, there is an induced map $f: X \rightarrow$ $\lim \operatorname{inv}\left(X_{n+1}, g_{n}\right)$. By Lemma 2.4, $f$ is one-to-one. Since all the maps $f_{n}$ are onto, also $f$ is a surjection. Thus $f$ is a homeomorphism.

\section{Examples and remarks.}

4.1. EXAMPLE. Let $Z$ denote a $\theta$-curve (i.e., $Z$ is a union of a circle and its diameter) and let $c, d$ denote the ramification points of $Z$. Let also $a$ and $b$ be any points of $Z-\{c, d\}$ which lie in distinct components of $Z-\{c, d\}$. Put $A^{\prime}=\{a, b, c, d\}$.

Let $Y=\{0,1\} \cup Z \times] 0,1\left[\right.$. Let $y$ be any point of $Y$. We define a family $B_{y}$ of basic neighbourhoods of $y$ in $Y$. If $y \in(Z-\{a, b\}) \times\{r\}$, for some $r \in] 0,1[$, then let $B_{y}$ be the family of all $V \times\{r\}$, where $V$ is an open subset of $Z$ such that $y \in V \times\{r\}$ and $a, b \notin V$. If $y=0$ then put

$$
B_{y}=\{\{y\} \cup Z \times] 0, r[: r \in] 0,1[\},
$$

and if $y=1$ then set

$$
B_{y}=\{\{y\} \cup Z \times] r, 1[: r \in] 0,1[\} .
$$

If $y=(a, r)$ for some $r \in] 0,1[$ then let

$B_{y}=\{Z \times] t, r[\cup V \times\{r\}: t \in] 0, r[, V$ is an open subset of $Z, a \in V$ and $b \notin V\}$, and if $y=(b, r)$ for some $r \in] 0,1[$ then let

$$
B_{y}=\{Z \times] r, t[\cup V \times\{r\}: t \in] r, 1[, V \text { is an open subset of } Z, b \in V \text { and } a \notin V\} \text {. }
$$

Put also $\left.A=\{0,1\} \cup A^{\prime} \times\right] 0,1$. It is obvious that $Y$ is a locally connected continuum which is a cyclic chain from 0 to 1 , each nondegenerate cyclic element of $Y$ is homeomorphic to $Z, A$ is a $T$-set in $Y$ which contains all cut points of $Y$, and each component of $Y-A$ is homeomorphic to $] 0,1[$. Define $f: Y \rightarrow[0,1]$ by the formulas $f(0)=0, f(1)=1$, and $f(z, r)=r$ for $z \in Z$ and $r \in] 0,1[$. Then $f$ is a continuous and monotone surjection.

Now, put $X_{1}=Z$ and $A_{1}^{1}=A^{\prime}$. Suppose that a locally connected continuum $X_{n}$ and a $T$-subset $A_{n}^{n}$ of $X_{n}$ are already constructed such that $X_{n}$ has no cut points and each component of $X_{n}-A_{n}^{n}$ is homeomorphic to ]0, 1 . 
Put $S_{n}=\left\{P: P\right.$ is a component of $\left.X_{n}-A_{n}^{n}\right\}$. If $P \in S_{n}$ then denote $\operatorname{bd}(P)=$ $\left\{e_{0}^{P}, e_{1}^{P}\right\}$ and let $f_{P}: \bar{P} \rightarrow[0,1]$ be any homeomorphism such that $f_{P}\left(e_{0}^{P}\right)=0$. Put $X_{n+1}^{\prime}=A_{n}^{n} \cup Y \times S_{n}$ and let $\rho$ be an equivalence relation on $X_{n+1}^{\prime}$ the only nondegenerate classes of which are of the form

$$
E_{i}^{P}=\left\{e_{i}^{P}\right\} \cup\left\{(i, Q) \in Y \times\{Q\}: Q \in S_{n} \text { and } e_{i}^{P} \in \operatorname{bd}(Q)\right\}
$$

for each $i \in\{0,1\}$ and $P \in S_{n}$ (note that the sets $E_{i}^{P}$ are finite).

Let $X_{n+1}$ denote the quotient set and $g: X_{n+1}^{\prime} \rightarrow X_{n+1}$ be the quotient function. Let $x$ be any point of $X_{n+1}$. We define a family $C_{x}$ of basic neighbourhoods of $x$ in $X_{n+1}$. If $x=g(y, P)$ for some $y \in Y-\{0,1\}$ and $P \in S_{n}$, then put $C_{x}=\{g(V \times\{P\}): V$ is open in $Y, y \in V$ and $0,1 \notin V\}$. If $x=g\left(x^{\prime}\right)$ for some $x^{\prime} \in A_{n}^{n}$ then set

$$
C_{x}=\left\{g\left(U \cap A_{n}^{n}\right) \cup \bigcup_{P \in S_{n}} g\left(f^{-1} f_{P}(U \cap P) \times\{P\}\right):\right.
$$

$U$ is an open neighbourhood of $x^{\prime}$ in $\left.X_{n}\right\}$.

Put

$$
A_{n}^{n+1}=g\left(A_{n}^{n}\right), \quad A_{n+1}^{n+1}=A_{n}^{n+1} \cup \bigcup_{P \in S_{n}} g(A \times\{P\})
$$

and let

$$
f_{n}(x)= \begin{cases}g^{-1}(x) \cap A_{n}^{n} & \text { if } x \in A_{n}^{n+1}, \\ f_{P}^{-1} f(y) & \text { if } x=g(y, P) \text { for some } y \in Y \text { and } P \in S_{n} .\end{cases}
$$

An easy proof shows that $X_{n+1}$ is a locally connected continuum without cut points, $A_{n}^{n+1}$ and $A_{n+1}^{n+1}$ are $T$-sets in $X_{n+1}$, and $f_{n}: X_{n+1} \rightarrow X_{n}$ is a $T$-map with respect to $A_{n}^{n+1}$. Moreover, $f_{n}$ is monotone.

Now, we inductively define $T$-subsets $A_{n}^{m}$ of $X_{m}$ for any positive integers $m, n$ so that $m \geq n$ : suppose that $A_{n}^{m}$ is already defined and put $A_{n}^{m+1}=A_{m}^{m+1} \cap f_{m}^{-1}\left(A_{n}^{m}\right)$.

Put $X=\lim \operatorname{inv}\left(X_{n+1}, f_{n}\right)$. Then $X$ is a locally connected continuum (see [1, Theorem 4.3, p. 241]) which has no cut points. For each positive integer $n$, set $A_{n}=\operatorname{liminv}\left(A_{n}^{m+1},\left.f_{m}\right|_{A_{n}^{m+1}}\right)$. Note that $A_{1}, A_{2}, \ldots$ is a sequence of $T$-subsets of $X$ which $T$-approximates $X$. Thus $X$ is a continuous image of some arc. It is not difficult to see that $r(X) \neq k$ for each nonnegative integer $k$.

Observe also that each point of $X$ has arbitrarily small open neighborhoods the boundary of which consists of either two or three points. Thus $X$ is a rim-finite continuum. This gives another proof that $X$ is a continuous image of some arc (see [15 and 18]). Moreover, note that $X$ fulfills the first axiom of countability.

4.2. REMARK. Let $X$ be a continuum without cut points which is a continuous image of some arc and let $A_{1}, A_{2}, \ldots$ be a sequence of $T$-subsets of $X$ which $T$ approximates $X$. One can use maps $f_{n}, g_{n}$ of Theorem 3.2 to find a directed by inclusion family $D$ of $T$-subsets of $X$ and maps $i_{A}: X \rightarrow X_{A}, j_{B}^{A}: X_{A} \rightarrow X_{B}$, for $A, B \in D, B \subset A$, such that

(a) each $i_{A}$ is a $T$-map with respect to $A$ and each $j_{B}^{A}$ is a $T$-map with respect to $i_{A}(B)$,

(b) each space $X_{A}$ is metrizable, 
(c) $T=\left(A, j_{A}^{B}, D\right)$ is an inverse system,

(d) $j_{B}^{A} \circ i_{A}=i_{B}$ for any $A, B \in D, B \subset A$, and

(e) $X$ is homeomorphic to $\lim \operatorname{inv} T$.

(Compare this with the proof given in [13]).

Recall that each locally connected continuum is an inverse limit of some inverse system of metrizable locally connected continua with monotone bonding mappings [8, Theorem 2].

4.3. EXAMPLE. Let $L$ denote the well-known long interval (see for example [3, p. 297]) and for each ordinal number $\alpha, 0<\alpha<\omega_{1}$, let $f_{\alpha}:[0,1] \times L \rightarrow[0,1] \times[0, \alpha]_{L}$ be a function defined by the formula

$$
f_{\alpha}(s, t)= \begin{cases}(s, t) & \text { if } t \leq_{L} \alpha \\ (s, \alpha) & \text { if } \alpha \leq_{L} t\end{cases}
$$

where $\leq_{L}$ is the natural ordering of $L$ from 0 to $\omega_{1}$. Moreover, if $\alpha, \beta$ are ordinals, $0<\alpha \leq \beta<\omega_{1}$, then put $f_{\alpha}^{\beta}=\left.f_{\alpha}\right|_{[0,1] \times[0, \beta]_{L}}$. Note that $f_{\alpha}, f_{\alpha}^{\beta}$ are monotone surjections and $f_{\alpha}^{\beta} \circ f_{\beta}^{\gamma}=f_{\alpha}^{\gamma}$ if $0<\alpha \leq \beta \leq \gamma<\omega_{1}$. Therefore $[0,1] \times L$ is homeomorphic to lim inv $\left([0,1] \times[0, \beta]_{L}, f_{\alpha}^{\beta}, \omega_{1}\right)$. Moreover, for each $0<\alpha<\omega_{1}$, $[0,1] \times[0, \alpha]_{L}$ is homeomorphic to the square $[0,1] \times[0,1]$, and so it is a continuous image of $[0,1]$. However, even in such a simple case as considered here, the inverse limit space $[0,1] \times L$ is not a continuous image of any arc or even any compact linearly ordered topological space $([\mathbf{2 2}]$, see also $[\mathbf{1 7}])$.

4.4. Problem. Suppose that $T=\left(X_{n+1}, f_{n}\right)$ is an inverse sequence such that all the spaces $X_{n}$ are continuous images of some arcs and all the mappings $f_{n}: X_{n+1} \rightarrow X_{n}$ are monotone surjections. Does it follow that the inverse limit space $X=\lim \operatorname{inv} T$ is a continuous image of any arc?

Recall that $X$ is locally connected ([1], see also [4]).

4.5. REMARK. In [12] it was also proved that if a continuum $X$ is a continuous image of some arc, then $X$ can be approximated by some family $J$ of finite dendrons (see $[19,17$ or 12] for the definition of the last notion). The proof given there can be simplified with the use of some ideas of the present paper. Namely, assume that $X$ is a continuum without cut points which is a continuous image of some arc and let $A_{1}, A_{2}, \ldots$ be a sequence of $T$-subsets of $X$ which $T$-approximates $X$. For each $n$ let $f_{n}: X \rightarrow X_{A_{n}}$ and $g_{n}: X_{A_{n+1}} \rightarrow X_{A_{n}}$ be maps constructed in Theorem 3.2. In [12] it was shown that, for each $n$, there is a family $J_{n}$ of finite dendrons in $X_{A_{n}}$ such that

(a) $J_{n}$ approximates $X_{A_{n}}$ and $X_{A_{n}}=\bigcup J_{n}$ (so for every points $x, y \in X_{A_{n}}$ we may define $[x, y]_{n}$ to be the unique subarc of $X_{A_{n}}$ such that $x, y$ are the endpoints of $[x, y]_{n}$ and $[x, y]_{n} \subset T$ for some $\left.T \in J_{n}\right)$, and

(b) there is a point $a \in A_{1}$ such that $g_{n}\left(\left[f_{n+1}(a), f_{n+1}(p)\right]_{n+1}\right)=\left[f_{n}(a), f_{n}(p)\right]_{n}$, for each $n$ and each point $p \in A_{n}$.

One can check that it is possible to choose maps $f_{n}$ and $g_{n}, n=1,2, \ldots$, in such a manner that $\left.g_{n+1}\right|_{\left[f_{n+1}(a), f_{n+1}(p)\right]_{n+1}}$ is monotone, for each point $p \in A_{n}$.

For any positive integers $n, k$ and points $b_{1}, \ldots, b_{k} \in A_{n}$ put

$$
D_{n}\left(b_{1}, \ldots, b_{k}\right)=\bigcup_{i=1}^{k}\left[f_{n}(a), f_{n}\left(b_{i}\right)\right]_{n}
$$


so $D_{n}\left(b_{1}, \ldots, b_{k}\right)$ is a finite dendron and

$$
\left.g_{n}\right|_{D_{n+1}\left(b_{1}, \ldots, b_{k}\right)}: D_{n+1}\left(b_{1}, \ldots, b_{k}\right) \rightarrow D_{n}\left(b_{1}, \ldots, b_{k}\right)
$$

is a monotone mapping. Put

$$
D\left(b_{1}, \ldots, b_{k}\right)=\operatorname{liminv}\left(D_{m+1}\left(b_{1}, \ldots, b_{k}\right),\left.g_{m}\right|_{D_{m+1}\left(b_{1}, \ldots, b_{k}\right)}, m \geq n\right) ;
$$

so $D\left(b_{1}, \ldots, b_{k}\right)$ is a finite dendron in $X$ (see for example [11]). Finally, put

$$
J=\left\{D\left(b_{1}, \ldots, b_{k}\right): k=1,2, \ldots, b_{1}, \ldots, b_{k} \in A_{n}, n=1,2, \ldots\right\} .
$$

It follows that $J$ is a family of finite dendrons which approximates $X$.

\section{REFERENCES}

1. C. E. Capel, Inverse limit spaces, Duke Math. J. 21 (1954), 233-245.

2. J. L. Cornette, Image of a Hausdorff arc is cyclically extensible and reducible, Trans. Amer. Math. Soc. 199 (1974), 255-267.

3. R. Engelking, General topology, PWN, 1977.

4. G. R. Gordh and S. Mardešc, Characterizing local connectedness in inverse limits, Pacific J. Math. 58 (1975), 411-417.

5. H. Hahn, Mengentheoretische Charakterisierung der stetigen Kurven, Sitzungsber. Akad. Wiss. Wien 123 (1914), 2433-2489.

6. J. G. Hocking and G. S. Young, Topology, Addison-Wesley, 1961.

7. S. Mardešic, On the Hahn-Mazurkiewicz theorem in non-metric spaces, Proc. Amer. Math. Soc. 11 (1960), 929-937.

8. __ Locally connected, ordered and chainable continua (in Serbo-Croatian, English summary), Rad Jugoslav. Akad. Znan. Umjet. Odjel Mat. Fiz. Tehn. Nauke 319 (1961), 147-166.

9. __ On the Hahn-Mazurkiewicz problem in non-metric spaces, General Topology and its Relations to Modern Analysis and Algebra II, Prague, 1966.

10. S. Mazurkiewicz, Sur les lignes de Jordan, Fund. Math. 1 (1920), 166-209.

11. J. van Mill and E. Wattel, Dendrons, Topology and Order Structures, part I, Math. Centre Tracts, vol. 142, Amsterdam, 1981, pp. 59-81.

12. J. Nikiel, Images of arcs-a nonseparable version of the Hahn-Mazurkiewicz theorem, Fund. Math. 129 (1988).

13. __ An elementary proof of a theorem of S. Mardesić, Proc. Amer. Math. Soc. 101 (1987), 385-386.

14. - A continuous partial ordering for images of arcs, Proc. Sixth Prague Topological Symp. 1986 (to appear).

15. B. J. Pearson, Mapping arcs and dendritic spaces onto netlike continua, Colloq. Math. 34 (1975), $39-48$.

16. L. B. Treybig, Arcwise connectivity in continuous images of ordered compacta, Glasnik Mat. 21 (1986), 201-211.

17. L. B. Treybig and L. E. Ward, The Hahn-Mazurkiewicz problem, Topology and Order Structures, part I, Math. Centre Tracts, vol. 142, Amsterdam, 1981, pp. 95-105.

18. L. E. Ward, The Hahn-Mazurkiewicz theorem for rim-finite continua, General Topology and Appl. 6 (1976), 183-190.

19. $\_$, A generalization of the Hahn-Mazurkiewicz theorem, Proc. Amer. Math. Soc. 58 (1976), 369-374.

20. G. T. Whyburn, Analytic topology, Amer. Math. Soc. Colloq. Publ., vol. 28, Amer. Math. Soc., Providence, R. I., 1942.

21. $ـ$ Cut points in general topological spaces, Proc. Nat. Acad. Sci. U.S.A. 61 (1968), 380-387.

22. G. S. Young, Representations of Banach spaces, Proc. Amer. Math. Soc. 13 (1962), 667-668.

Institute of Mathematics, University of Wroclaw, Pl. GRunWaldiki 2/4, 50-384 WROCLAW, POLAND 